\title{
Classification of commutative association schemes with almost commutative Terwilliger algebras
}

\author{
Rie Tanaka
}

Received: 6 January 2010 / Accepted: 31 March 2010 / Published online: 21 April 2010

(C) Springer Science+Business Media, LLC 2010

\begin{abstract}
We classify the commutative association schemes such that all nonprimary irreducible modules of their Terwilliger algebras are one-dimensional.
\end{abstract}

Keywords Association scheme · Terwilliger algebra · Wreath product · Poset

\section{Introduction}

The Terwilliger algebra $\mathcal{T}=\mathcal{T}(x)$ of an association scheme $\mathcal{X}=\left(X,\left\{R_{i}\right\}_{0 \leq i \leq d}\right)$ $(x \in X)$ is introduced in [9]. The algebra $\mathcal{T}$ is a semisimple $\mathbb{C}$-algebra, and not commutative if $|X|>1$. It is important to determine the irreducible $\mathcal{T}$-modules for each $\mathcal{X}$ and consider what combinatorial information on $\mathcal{X}$ can be deduced from a property of its irreducible $\mathcal{T}$-modules.

In [4], the irreducible $\mathcal{T}$-modules of a wreath product of 1-class association schemes are determined and it is shown that every non-primary irreducible $\mathcal{T}$-module is 1-dimensional. A semisimple algebra is commutative if all of its irreducible modules are 1-dimensional. So we may say $\mathcal{T}$ is almost commutative if all of its nonprimary irreducible $\mathcal{T}$-modules are 1-dimensional. In this paper, we classify the commutative association schemes whose Terwilliger algebras are almost commutative.

In the classification, we first characterize such association schemes in terms of intersection numbers $p_{i j}^{h}$ and Krein parameters $q_{i j}^{h}$ of $\mathcal{X}$. In the study of association schemes, $p_{i j}^{h}$ and $q_{i j}^{h}$ play important roles. By considering vanishing conditions of $p_{i j}^{h}, q_{i j}^{h}$, several important classes of association schemes such as $P$-polynomial and $Q$-polynomial schemes have been introduced [1,3]. In this paper, we introduce a class of association schemes whose intersection numbers vanish in a drastic way.

R. Tanaka $(\bowtie)$

Graduate School of Information Sciences, Tohoku University, Aramaki Aza-Aoba, Aoba-ku, Sendai 980-8579, Japan

e-mail: hrie@math.is.tohoku.ac.jp 
(B) For all distinct $h, i$, there is exactly one $j$ such that $p_{i j}^{h} \neq 0(0 \leq h, i, j \leq d)$.

$\left(\mathrm{B}^{*}\right)$ For all distinct $h, i$, there is exactly one $j$ such that $q_{i j}^{h} \neq 0(0 \leq h, i, j \leq d)$.

The following is the main theorem of this paper:

Theorem 1 Let $\mathcal{X}=\left(X,\left\{R_{i}\right\}_{0 \leq i \leq d}\right)$ be a commutative association scheme. The following are equivalent:

(i) Every non-primary irreducible $\mathcal{T}(x)$-module is 1-dimensional for some $x \in X$.

(ii) Every non-primary irreducible $\mathcal{T}(x)$-module is 1-dimensional for all $x \in X$.

(iii) The intersection numbers of $\mathcal{X}$ satisfy (B).

(iv) The Krein parameters of $\mathcal{X}$ satisfy $\left(\mathrm{B}^{*}\right)$.

(v) $\mathcal{X}$ is a wreath product of association schemes $\mathcal{X}_{1}, \mathcal{X}_{2}, \ldots, \mathcal{X}_{n}$ where each $\mathcal{X}_{i}$ is either a 1-class association scheme or the group scheme of a finite abelian group.

Moreover, if (i)-(v) hold, $\mathcal{X}$ is triply regular.

The paper is organized as follows: Sect. 2 reviews some basic properties of association schemes and their Terwilliger algebras. In the proof of Theorem 1, we will adopt the following strategy. In Sect. 3, we will prove the equivalence of assertions (i)-(iv) from the theorem. In Sect. 4, we will prove the equivalence of assertions (iii) and (v) from the theorem.

\section{Preliminaries}

For general introduction to association schemes and Terwilliger algebras, we refer the reader to $[1,3,6,9]$.

Let $X$ be a finite nonempty set and $\operatorname{Mat}_{X}(\mathbb{C})$ the set of square complex matrices whose entries are indexed by the elements of $X$. Let $R_{0}, R_{1}, \ldots, R_{d}$ be nonempty subsets of $X \times X$, and for each $0 \leq i \leq d$, let $A_{i} \in \operatorname{Mat}_{X}(\mathbb{C})$ be the adjacency matrix of $R_{i}$ :

$$
\left(A_{i}\right)_{x y}= \begin{cases}1 & \text { if }(x, y) \in R_{i}, \\ 0 & \text { otherwise. }\end{cases}
$$

The pair $\mathcal{X}=\left(X,\left\{R_{i}\right\}_{0 \leq i \leq d}\right)$ is called a $d$-class association scheme if the following (AS1)-(AS4) hold:

(AS1) $A_{0}=I$, the identity matrix.

(AS2) $A_{0}+A_{1}+\cdots+A_{d}=J$, the all-one's matrix.

(AS3) For $0 \leq i \leq d$, there is $0 \leq i^{\prime} \leq d$ such that $A_{i^{\prime}}=A_{i}^{T}$, the transpose of $A_{i}$.

(AS4) $A_{i} A_{j}$ is a linear combination of $A_{0}, A_{1}, \ldots, A_{d}$ for $0 \leq i, j \leq d$.

By (AS1) and (AS4), $\mathcal{M}:=\operatorname{Span}\left\{A_{0}, A_{1}, \ldots, A_{d}\right\}$ is a subalgebra of $\operatorname{Mat}_{X}(\mathbb{C})$; this is the Bose-Mesner algebra of $\mathcal{X}$. By (AS2), the $A_{i}$ are linearly independent and thus form a basis for $\mathcal{M}$. We say $\mathcal{X}$ is commutative if $\mathcal{M}$ is commutative. 
Throughout the paper, we assume $\mathcal{X}$ is commutative. By (AS3), $\mathcal{M}$ is closed under conjugate-transposition. Hence $\mathcal{M}$ is a commutative semisimple algebra and has a basis $\left\{E_{i}: 0 \leq i \leq d\right\}$ of primitive idempotents:

$$
\begin{aligned}
& E_{i} E_{j}=\delta_{i j} E_{i} ; \\
& E_{0}+E_{1}+\cdots+E_{d}=I ;
\end{aligned}
$$

where we always set $E_{0}=|X|^{-1} J$. Note that for each $0 \leq i \leq d$, there is $0 \leq \hat{i} \leq d$ such that $E_{\hat{i}}=E_{i}^{T}$. It also follows from (AS2) that $\mathcal{M}$ is closed under entry-wise multiplication denoted o.

The intersection numbers $p_{i j}^{h}$ and the Krein parameters $q_{i j}^{h}$ are defined by the following equations:

$$
A_{i} A_{j}=\sum_{h=0}^{d} p_{i j}^{h} A_{h}, \quad E_{i} \circ E_{j}=|X|^{-1} \sum_{h=0}^{d} q_{i j}^{h} E_{h} \quad(0 \leq i, j \leq d) .
$$

Let $R_{i}(x):=\left\{y \in X:(x, y) \in R_{i}\right\} \quad(x \in X, 0 \leq i \leq d)$. By (3), $p_{i j}^{h}=\mid R_{i}(x) \cap$ $R_{j^{\prime}}(y) \mid$ where $(x, y) \in R_{h}(x, y \in X)$. Clearly, the $p_{i j}^{h}$ are non-negative integers. In particular, $k_{i}:=p_{i i^{\prime}}^{0}=\left|R_{i}(x)\right| \neq 0$. We can also verify that the $q_{i j}^{h}$ are non-negative real numbers and $m_{i}:=q_{i \hat{i}}^{0}=\operatorname{tr} E_{i} \neq 0[1,6]$. Note that

$$
\begin{aligned}
k_{i} & =\sum_{h=0}^{d} p_{i h}^{j} \quad(0 \leq i, j \leq d) ; \\
p_{i j}^{h} & =0 \quad \Longleftrightarrow \quad p_{h j^{\prime}}^{i}=0 \quad \Longleftrightarrow \quad p_{i^{\prime} h}^{j}=0 ; \\
p_{i j}^{h} & =p_{j i}^{h} .
\end{aligned}
$$

Fix $x \in X$. For each $0 \leq i \leq d$, let $E_{i}^{*}=E_{i}^{*}(x), A_{i}^{*}=A_{i}^{*}(x)$ be the diagonal matrices in $\operatorname{Mat}_{X}(\mathbb{C})$ with $y y$-entries $\left(E_{i}^{*}\right)_{y y}=\left(A_{i}\right)_{x y},\left(A_{i}^{*}\right)_{y y}=|X|\left(E_{i}\right)_{x y}$. By definition, we have the following:

$$
\begin{aligned}
& E_{i}^{*} E_{j}^{*}=\delta_{i j} E_{i}^{*} \quad(0 \leq i, j \leq d) ; \\
& E_{0}^{*}+E_{1}^{*}+\cdots+E_{d}^{*}=I .
\end{aligned}
$$

We also have the following:

$$
\begin{aligned}
A_{0}^{*} & =I ; \\
A_{0}^{*}+A_{1}^{*}+\cdots+A_{d}^{*} & =|X| E_{0}^{*} \\
A_{i}^{*} A_{j}^{*} & =\sum_{h=0}^{d} q_{i j}^{h} A_{h}^{*} \quad(0 \leq i, j \leq d) .
\end{aligned}
$$

The $E_{i}^{*}$ and the $A_{i}^{*}$ form two bases for the dual Bose-Mesner algebra $\mathcal{M}^{*}=$ $\mathcal{M}^{*}(x)$ with respect to $x$. The Terwilliger algebra $\mathcal{T}=\mathcal{T}(x)$ of $\mathcal{X}$ with respect to 
$x$ is the subalgebra of $\operatorname{Mat}_{X}(\mathbb{C})$ generated by $\mathcal{M}$ and $\mathcal{M}^{*}$. Since $\mathcal{T}$ is closed under conjugate-transposition, it is semisimple. It is easily shown that $\mathcal{T}$ is not commutative if $|X|>1$. We can verify the following:

\section{Lemma 2 ([9])}

(i) $E_{i}^{*} A_{j} E_{h}^{*}=0$ if and only if $p_{i j}^{h}=0$.

(ii) $E_{i} A_{j}^{*} E_{h}=0$ if and only if $q_{i j}^{h}=0$.

Nonzero matrices among $E_{i}^{*} A_{j} E_{h}^{*}(0 \leq h, i, j \leq d)$ form a basis of $\mathcal{M}^{*} \mathcal{M} \mathcal{M}^{*}$, and $\mathcal{T}$ is generated by $\mathcal{M}^{*} \mathcal{M} \mathcal{M}^{*}$. When $\mathcal{T}$ coincides with $\mathcal{M}^{*} \mathcal{M} \mathcal{M}^{*}, \mathcal{X}$ has a special regularity:

Definition $3 \mathcal{X}$ is called triply regular if for all $0 \leq h, i, j, l, m, n \leq d$, the size

$$
\left|R_{h}(x) \cap R_{i}(y) \cap R_{j}(z)\right|
$$

is independent of $x, y, z \in X$ where $(x, y) \in R_{l},(y, z) \in R_{m},(x, z) \in R_{n}$.

Proposition 4 ([7]) $\mathcal{X}$ is triply regular if and only if $\mathcal{T}(x)=\mathcal{M}^{*}(x) \mathcal{M M}^{*}(x)$ for all $x \in X$.

The triple-regularity was first introduced in the study of spin models (see [5]).

Let $\mathbb{C}^{X}$ be the set of the complex column vectors with coordinates indexed by $X$, and observe that $\operatorname{Mat}_{X}(\mathbb{C})$ acts on $\mathbb{C}^{X}$ from the left. We equip $\mathbb{C}^{X}$ with the Hermitian inner product $\langle$,$\rangle . For y \in X$, let $\hat{y}$ be the vector in $\mathbb{C}^{X}$ with a 1 in coordinate $y$ and 0 elsewhere. Then $\{\hat{y}: y \in X\}$ forms an orthonormal basis of $\mathbb{C}^{X}$.

For a $\mathcal{T}$-module $W$, its orthogonal complement $W^{\perp}$ is also a $\mathcal{T}$-module since $\mathcal{T}$ is closed under conjugate-transposition. Thus $\mathbb{C}^{X}$ is decomposed as a direct sum of irreducible $\mathcal{T}$-modules. Let $\mathbf{1}:=\sum_{y \in X} \hat{y} \in \mathbb{C}^{X}$.

Lemma 5 ([9]) The space $\operatorname{Span}\left\{E_{i}^{*} \mathbf{1}: 0 \leq i \leq d\right\}=\operatorname{Span}\left\{E_{i} \hat{x}: 0 \leq i \leq d\right\}$ is an irreducible $\mathcal{T}(x)$-module of dimension $d+1$.

The $\mathcal{T}$-module in Lemma 5 will be denoted $W_{0}=W_{0}(x)$. This $\mathcal{T}$-module $W_{0}$ is said to be primary.

Definition 6 Let $\mathcal{X}_{1}=\left(X_{1},\left\{R_{i}^{(1)}\right\}_{0 \leq i \leq d_{1}}\right), \mathcal{X}_{2}=\left(X_{2},\left\{R_{i}^{(2)}\right\}_{0 \leq i \leq d_{2}}\right)$ be association schemes, and $A_{i}^{(1)}\left(0 \leq i \leq d_{1}\right), A_{i}^{(2)}\left(0 \leq i \leq d_{2}\right)$ their adjacency matrices. Let $X=$ $X_{1} \times X_{2}$ and $R_{i}\left(0 \leq i \leq d_{1}+d_{2}\right)$ be subsets of $X \times X$ whose adjacency matrices $A_{i}$ are defined as follows:

$$
\begin{aligned}
A_{i} & =A_{i}^{(1)} \otimes A_{0}^{(2)} \quad\left(0 \leq i \leq d_{1}\right), \\
A_{d_{1}+i} & =J \otimes A_{i}^{(2)} \quad\left(1 \leq i \leq d_{2}\right),
\end{aligned}
$$

where $\otimes$ denotes the Kronecker product. Then $\left(X,\left\{R_{i}\right\}_{0 \leq i \leq d_{1}+d_{2}}\right)$ is an association scheme; this is called the wreath product of $\mathcal{X}_{1}$ and $\mathcal{X}_{2}$, denoted $\mathcal{X}_{1}<\mathcal{X}_{2}$. 
Definition 7 Let $\mathcal{P}, \mathcal{Q}$ be partially ordered sets (posets). The ordinal sum $\mathcal{P} \oplus \mathcal{Q}$ of $\mathcal{P}$ and $\mathcal{Q}$ is the poset on the union $\mathcal{P} \cup \mathcal{Q}$ such that $x \prec y$ in $\mathcal{P} \oplus \mathcal{Q}$ if (a) $x \prec y$ in $\mathcal{P}$, or (b) $x \prec y$ in $\mathcal{Q}$, or (c) $x \in \mathcal{P}$ and $y \in \mathcal{Q}$.

Basic concepts and properties of posets can be found in [8, Chap. 3].

\section{Characterization}

In this section, we prove the equivalence of (i)-(iv) in Theorem 1. Fix $x \in X$. Let $\mathcal{T}=\mathcal{T}(x)$ be the Terwilliger algebra of $\mathcal{X}$ with respect to $x$.

Lemma 8 The following are equivalent:

(i) $\mathbb{C}^{X}=W_{0}$.

(ii) $k_{i}=1$ for all $0 \leq i \leq d$.

(iii) $m_{i}=1$ for all $0 \leq i \leq d$.

(iv) $\mathcal{X}$ is the group association scheme of a finite abelian group.

Moreover, if (i)-(iv) hold, then (B) and ( $\left.\mathrm{B}^{*}\right)$ hold.

Proof Since $1=\operatorname{dim} E_{i}^{*} W_{0} \leq \operatorname{dim} E_{i}^{*} \mathbb{C}^{X}=k_{i}$, we have (i) $\Leftrightarrow$ (ii). Similarly, we have (i) $\Leftrightarrow$ (iii). Obviously, $\mathcal{M}$ is a group algebra if and only if (ii) holds. Since $\mathcal{X}$ is commutative, we have (ii) $\Leftrightarrow$ (iv). The last assertion is clear.

See $[1,2]$, for details of group association schemes.

Lemma 9 The following hold.

(i) (B) holds if and only if $E_{i}^{*} A_{j} E_{h}^{*}=|X| E_{i}^{*} E_{0} E_{h}^{*}$ for $0 \leq h, i, j \leq d$ with $h \neq i$ and $p_{i j}^{h} \neq 0$.

(ii) (B*) holds if and only if $E_{i} A_{j}^{*} E_{h}=|X| E_{i} E_{0}^{*} E_{h}$ for $0 \leq h, i, j \leq d$ with $h \neq i$ and $q_{i j}^{h} \neq 0$.

Proof (i) Note that by (AS2), $|X| E_{i}^{*} E_{0} E_{h}^{*}=E_{i}^{*} A_{j} E_{h}^{*}+\sum_{l \neq j} E_{i}^{*} A_{l} E_{h}^{*}$. Suppose (B) holds. Let $p_{i j}^{h} \neq 0$ and $h \neq i$. By Lemma 2 and (B), $E_{i}^{*} A_{l} E_{h}^{*} \neq 0$ only if $l=j$. Thus $|X| E_{i}^{*} E_{0} E_{h}^{*}=E_{i}^{*} A_{j} E_{h}^{*}$. Conversely, suppose $E_{i}^{*} A_{j} E_{h}^{*}=|X| E_{i}^{*} E_{0} E_{h}^{*}$. Then we have $\sum_{l \neq j} E_{i}^{*} A_{l} E_{h}^{*}=0$. Since the $E_{i}^{*} A_{l} E_{h}^{*}$ are linearly independent, $E_{i}^{*} A_{l} E_{h}^{*}=$ 0 if $l \neq j$, that is, $p_{i l}^{h} \neq 0$ only if $l=j$. We obtain (B). (ii) By exchanging the roles of the $p_{i j}^{h}, E_{i}^{*}, A_{i},(\mathrm{AS} 2)$ by those of the $q_{i j}^{h}, E_{i}, A_{i}^{*},(10)$, we obtain the assertion.

Proposition 10 If every non-primary irreducible $\mathcal{T}$-module is 1-dimensional, then (B), ( $\left.\mathrm{B}^{*}\right)$ hold.

Proof By Lemma 8, if $\mathbb{C}^{X}=W_{0}$, (B), (B*) hold. Suppose $\mathbb{C}^{X} \neq W_{0}$. Since the nontrivial subspaces $E_{h}^{*} W_{0}^{\perp}$ are direct sums of non-primary irreducible $\mathcal{T}$-modules, they are $\mathcal{T}$-modules. Hence $E_{i}^{*} A_{j} E_{h}^{*} W_{0}^{\perp}=0$ if $h \neq i$. Let $p_{i j}^{h} \neq 0$ and $h \neq i$. Let 
$u_{2}, \ldots, u_{k_{h}}$ be a basis of $E_{h}^{*} W_{0}^{\perp}$. Then $E_{h}^{*} \mathbf{1}, u_{2}, \ldots, u_{k_{h}}$ form a basis of $E_{h}^{*} \mathbf{C}^{X}$. We have $E_{i}^{*} A_{j} E_{h}^{*} \mathbf{1}=p_{i j}^{h} E_{i}^{*} \mathbf{1}$ and $|X| E_{i}^{*} E_{0} E_{h}^{*} \mathbf{1}=k_{h} E_{i}^{*} \mathbf{1}$. Clearly, $E_{i}^{*} A_{j} E_{h}^{*} u_{l}=$ $|X| E_{i}^{*} E_{0} E_{h}^{*} u_{l}=0\left(2 \leq l \leq k_{h}\right)$. Hence $k_{h} \cdot E_{i}^{*} A_{j} E_{h}^{*}=p_{i j}^{h} \cdot|X| E_{i}^{*} E_{0} E_{h}^{*}$. Since $|X| E_{i}^{*} E_{0} E_{h}^{*}=\sum_{l=0}^{d} E_{i}^{*} A_{l} E_{h}^{*}$ and the $E_{i}^{*} A_{l} E_{h}^{*}$ are linearly independent, $k_{h}=p_{i j}^{h}$ and thus $E_{i}^{*} A_{j} E_{h}^{*}=|X| E_{i}^{*} E_{0} E_{h}^{*}$. By Lemma 9, (B) holds. Similarly, exchanging the roles of $p_{i j}^{h}, E_{i}^{*}, A_{i}, \mathbf{1}$ by those of the $q_{i j}^{h}, E_{i}, A_{i}^{*}, \hat{x},\left(\mathrm{~B}^{*}\right)$ holds.

Lemma 11 The following hold.

(i) If (B) holds, then $E_{i}^{*} \mathcal{M} E_{i}^{*}$ is a commutative algebra for $0 \leq i \leq d$.

(ii) If ( $\left.\mathrm{B}^{*}\right)$ holds, then $E_{i} \mathcal{M}^{*} E_{i}$ is a commutative algebra for $0 \leq i \leq d$.

Proof (i) By (7), (8) and Lemma 9, for $h, j(0 \leq h, j \leq d)$ we have

$$
\begin{aligned}
E_{i}^{*} A_{h} A_{j} E_{i}^{*} & =E_{i}^{*} A_{h}\left(\sum_{l=0}^{d} E_{l}^{*}\right) A_{j} E_{i}^{*}=E_{i}^{*} A_{h} E_{i}^{*} A_{j} E_{i}^{*}+\sum_{l \neq i} E_{i}^{*} A_{h} E_{l}^{*} A_{j} E_{i}^{*} \\
& =E_{i}^{*} A_{h} E_{i}^{*} \cdot E_{i}^{*} A_{j} E_{i}^{*}+\sum_{l \neq i} E_{i}^{*} A_{h} E_{l}^{*} \cdot E_{l}^{*} A_{j} E_{i}^{*} \\
& =E_{i}^{*} A_{h} E_{i}^{*} \cdot E_{i}^{*} A_{j} E_{i}^{*}+\sum_{l \neq i, p_{i h}^{l} \neq 0, p_{l j}^{i} \neq 0}|X|^{2} E_{i}^{*} E_{0} E_{l}^{*} E_{0} E_{i}^{*} \\
& =E_{i}^{*} A_{h} E_{i}^{*} \cdot E_{i}^{*} A_{j} E_{i}^{*}+\sum_{l \neq i, p_{i h}^{l} \neq 0, p_{l j}^{i} \neq 0} k_{l}|X| E_{i}^{*} E_{0} E_{i}^{*} .
\end{aligned}
$$

Therefore

$$
\begin{aligned}
E_{i}^{*} A_{h} E_{i}^{*} \cdot E_{i}^{*} A_{j} E_{i}^{*} & =E_{i}^{*} A_{h} A_{j} E_{i}^{*}-\sum_{l \neq i, p_{i h}^{l} \neq 0, p_{l j}^{i} \neq 0} k_{l}|X| E_{i}^{*} E_{0} E_{i}^{*} \\
& =\sum_{l=0}^{d} p_{h j}^{l} E_{i}^{*} A_{l} E_{i}^{*}-\sum_{l \neq i, p_{i h}^{l} \neq 0, p_{l j}^{i} \neq 0} k_{l}|X| E_{i}^{*} E_{0} E_{i}^{*} .
\end{aligned}
$$

Hence $E_{i}^{*} \mathcal{M} E_{i}^{*}$ is closed under multiplication. Since $\mathcal{M}$ is commutative, by (12), $E_{i}^{*} \mathcal{M} E_{i}^{*}$ is commutative as well. (ii) Exchanging the roles of the $p_{i j}^{h}, E_{i}^{*}, A_{i}, \mathcal{M}$, (7), (8) by those of the $q_{i j}^{h}, E_{i}, A_{j}^{*}, \mathcal{M}^{*},(1),(2)$, the assertion holds.

Lemma 12 The following hold.

(i) If (B) holds, then $\mathcal{T}=\mathcal{M}^{*} \mathcal{M} \mathcal{M}^{*}$.

(ii) If ( $\left.\mathrm{B}^{*}\right)$ holds, then $\mathcal{T}=\mathcal{M} \mathcal{M}^{*} \mathcal{M}$.

Proof (i) Let $U:=\operatorname{Span}\left\{E_{i}^{*} E_{0} E_{h}^{*}: h \neq i, 0 \leq h, i \leq d\right\}$. By Lemma 9, $U=$ $\operatorname{Span}\left\{E_{i}^{*} A_{j} E_{h}^{*}: h \neq i, 0 \leq h, i, j \leq d\right\}$. Hence $\mathcal{M}^{*} \mathcal{M} \mathcal{M}^{*}=\left(\bigoplus_{i=0}^{d} E_{i}^{*} \mathcal{M} E_{i}^{*}\right) \oplus U$ 
(a direct sum). By Lemma 11 and calculations in its proof, the $E_{i}^{*} \mathcal{M} E_{i}^{*}$ and $U$ are closed under multiplication. We can easily verify that the $E_{i}^{*} \mathcal{M} E_{i}^{*}$ act on $U$ from both the left and the right. Thus $\mathcal{M}^{*} \mathcal{M} \mathcal{M}^{*}$ forms an algebra, i.e., $\mathcal{T}=\mathcal{M}^{*} \mathcal{M} \mathcal{M}^{*}$. (ii) Exchanging the roles of the $p_{i j}^{h}, E_{i}^{*}, A_{i}, \mathcal{M}$ by those of the $q_{i j}^{h}, E_{i}, A_{i}^{*}, \mathcal{M}^{*}$, the assertion holds.

Corollary 13 If $(\mathrm{B})$ holds, $\mathcal{X}$ is triply regular.

Proof By Proposition 4 and Lemma 12, the assertion holds.

Proposition 14 The following hold.

(i) If (B) holds, every non-primary irreducible $\mathcal{T}$-module is 1-dimensional.

(ii) If ( $\left.\mathrm{B}^{*}\right)$ holds, every non-primary irreducible $\mathcal{T}$-module is 1-dimensional.

Proof (i) By Lemma $11, E_{i}^{*} \mathcal{M} E_{i}^{*}$ is a commutative semisimple algebra. Hence $E_{i}^{*} \mathbb{C}^{X}$ is decomposed as the direct sum of the common eigenspaces of $E_{i}^{*} \mathcal{M} E_{i}^{*}$. Assume $\mathbb{C}^{X} \neq W_{0}$. Let $u \in\left(E_{i}^{*} \mathbb{C}^{X}\right) \cap W_{0}^{\perp}$ be a common eigenvector of $E_{i}^{*} \mathcal{M} E_{i}^{*}$. Since $\operatorname{Span}\{u\}$ is closed under the action of $\mathcal{M}^{*} \mathcal{M} \mathcal{M}^{*}$, by Lemma 12 , it is a $\mathcal{T}$ module. In this way, $\left(E_{i}^{*} \mathbb{C}^{X}\right) \cap W_{0}^{\perp}$ is decomposed as a direct sum of 1-dimensional irreducible $\mathcal{T}$-modules. The assertion is clear. (ii) Exchanging the roles of the $E_{i}^{*}, \mathcal{M}$ by those of the $E_{i}, \mathcal{M}^{*}$, the assertion holds.

Proof of Theorem 1 (i) $\Leftrightarrow$ (ii) $\Leftrightarrow$ (iii) $\Leftrightarrow$ (iv) By Proposition 10, we have (i) $\Rightarrow$ (iii) and (i) $\Rightarrow$ (iv). By Proposition 14, we have (iii) $\Rightarrow$ (ii) and (iv) $\Rightarrow$ (ii). Obviously, (ii) $\Rightarrow$ (i).

\section{Classification}

In this section, we prove (iii) $\Leftrightarrow$ (v) in Theorem 1 . Throughout the section, let $\mathcal{X}=$ $\left(X,\left\{R_{i}\right\}_{0 \leq i \leq d}\right)$ be a commutative association scheme whose intersection numbers satisfy (B).

Lemma 15 If $p_{i j}^{j} \neq 0$ for $1 \leq i, j \leq d$, then $p_{i^{\prime} j}^{j} \neq 0, p_{i j^{\prime}}^{j^{\prime}} \neq 0$ and $p_{i^{\prime} j^{\prime}}^{j^{\prime}} \neq 0$.

Proof By (5), $p_{i^{\prime} j}^{j} \neq 0$ and $p_{j j^{\prime}}^{i} \neq 0$. Again by (5), $p_{j^{\prime} i}^{j^{\prime}} \neq 0$, and by (6) we have $p_{i j^{\prime}}^{j^{\prime}} \neq 0$. Again by (5), $p_{i^{\prime} j^{\prime}}^{j^{\prime}} \neq 0$.

Lemma 16 If $p_{i^{\prime} j}^{j} \neq 0$ for $1 \leq i, j \leq d$ with $i^{\prime} \neq j$, then $A_{i} A_{j}=p_{i j}^{j} A_{j}$.

Proof By (B), $p_{i^{\prime} l}^{j}=0$ if $l \neq j$. By (5), $p_{i^{\prime} l}^{j}=0$ if and only if $p_{i j}^{l}=0$. Hence $p_{i j}^{l}=0$ if $l \neq j$, and the assertion holds.

$$
\text { Let }[i]=\left[i^{\prime}\right]:=\left\{i, i^{\prime}\right\} \text { for } 1 \leq i \leq d \text {. }
$$


Lemma 17 Let $\prec$ be the relation on $\{[i]: 1 \leq i \leq d\}$ defined as follows: $[i] \prec[j]$ if $[i]=[j]$ or $p_{i j}^{j} \neq 0$. Then $\prec$ is a partial order.

Proof By Lemma 15, the relation $\prec$ is well-defined. By definition, $\prec$ is reflexive. Suppose $[i] \prec[j]$ and $[j] \prec[i]$. Moreover suppose $p_{i j}^{j} \neq 0$ and $p_{j i}^{i} \neq 0$. Since $p_{i j}^{j} \neq 0$, by (5), $p_{j j^{\prime}}^{i} \neq 0$. If $i \neq j$, by (B), $p_{j l}^{i} \neq 0$ only if $l=j^{\prime}$. Since $p_{j i}^{i} \neq 0$, $i=j^{\prime}$. Thus $[i]=[j]$ and $\prec$ is antisymmetric. Suppose $[h] \prec[i]$ and $[i] \prec[j]$ for distinct $[h],[i],[j]$. Since $p_{h^{\prime} i}^{i} \neq 0, p_{i^{\prime} j}^{j} \neq 0$ and $h^{\prime} \neq i, i^{\prime} \neq j$, by Lemma 16, $\left(A_{h} A_{i}\right) A_{j}=p_{h i}^{i} A_{i} A_{j}=p_{h i}^{i} p_{i j}^{j} A_{j} \neq 0$. On the other hand, $A_{h}\left(A_{i} A_{j}\right)=p_{i j}^{j} A_{h} A_{j}$. Therefore $A_{h} A_{j}=p_{h j}^{j} A_{j} \neq 0$ and $p_{h j}^{j} \neq 0$. Hence $[h] \prec[j]$, and thus $\prec$ is transitive.

For the rest of the section, let $\mathcal{P}:=(\{[i]: 1 \leq i \leq d\}, \prec)$ be the poset defined in Lemma 17.

Lemma 18 Let $[i]$ be a minimal element of $\mathcal{P}$. If $k_{i} \geq 2$, then $[i]$ is the minimum.

Proof Suppose $k_{i} \geq 2$. Let $x \in X$. Take distinct $y, z \in R_{i}(x)$. Since [i] is minimal, $(y, z) \in R_{i} \cup R_{i^{\prime}}$. Let $[j] \in \mathcal{P} \backslash\{[i]\}$ and $w \in R_{j}(x)$. Since $i \neq j$, by (B), $y, z \in$ $R_{h}(w)$ for some $1 \leq h \leq d$. Hence $p_{h i}^{h}=p_{i h}^{h} \neq 0$, and [i] $\prec[h]$. If $[h]=[i]$, by the configuration of $x, y, w$, we have $p_{j i}^{i} \neq 0$ or $p_{j i^{\prime}}^{i^{\prime}} \neq 0$, i.e., $[j] \prec[i]$, contradicting [i] is minimal. Hence $[h] \neq[i]$. By the configuration of $x, w, y, p_{j h}^{i}=p_{h j}^{i} \neq 0$. Since $[h] \neq[i]$ and $p_{h h^{\prime}}^{i} \neq 0, h^{\prime}=j$. So $[h]=[j]$. Since $[i] \prec[h]$, we have $[i] \prec[j]$.

Lemma 19 The following hold.

(i) If $\mathcal{P}$ is a singleton, then either $d=1$ or $\mathcal{X}$ is the group association scheme of $\mathbb{Z}_{3}$.

(ii) If $\mathcal{P}$ is an antichain with at least 2 elements, $\mathcal{X}$ is the group association scheme of a finite abelian group.

Proof (i) Let $\mathcal{P}=\{[i]\}$. Suppose $k_{i} \geq 2$. Then $p_{i i}^{i} \neq 0$. By (5), (6), we have $p_{i^{\prime} i}^{i}=$ $p_{i i^{\prime}}^{i} \neq 0$. If $i \neq i^{\prime}$, by (B), (4), $k_{i^{\prime}}=\sum_{l=0}^{d} p_{i^{\prime} l}^{i}=p_{i^{\prime} i}^{i}=p_{i i^{\prime}}^{i}<p_{i 0}^{i}+p_{i i^{\prime}}^{i} \leq \sum_{l=0}^{d} p_{i l}^{i}=$ $k_{i}$, contradicting $k_{i^{\prime}}=k_{i}$. Hence $i=i^{\prime}$, i.e., $d=1$. Suppose $d \neq 1$. Then $k_{i}=k_{i^{\prime}}=1$ and by Lemma $8, \mathcal{X}$ is the group association scheme of $\mathbb{Z}_{3}$. (ii) By Lemma $18, k_{i}=1$ for all $[i] \in \mathcal{P}$. By Lemma 8 , the assertion holds.

Lemma 20 Suppose $\mathcal{P}=\mathcal{P}_{1} \oplus \mathcal{P}_{2}$ for some subposets $\mathcal{P}_{1}, \mathcal{P}_{2}$ of $\mathcal{P}$. Then $R_{0} \cup$ $\left(\bigcup_{[i] \in \mathcal{P}_{1}} R_{i}\right)$ is an equivalence relation on $X$, i.e., $\mathcal{X}$ is imprimitive. Moreover, $A_{i}$ $(1 \leq i \leq d)$ can be written as follows:

$$
\begin{array}{ll}
A_{i}=B_{i} \otimes I_{q} & \left([i] \in \mathcal{P}_{1}\right) \\
A_{i}=J_{p} \otimes C_{i} & \left([i] \in \mathcal{P}_{2}\right) ;
\end{array}
$$


where $B_{0}=I_{p}, B_{i}\left([i] \in \mathcal{P}_{1}\right)$ are the adjacency matrices of the subscheme $\mathcal{X}_{1}$ and $C_{0}=I_{q}, C_{i}\left([i] \in \mathcal{P}_{2}\right)$ are those of the quotient scheme $\mathcal{X}_{2}=\mathcal{X} / \mathcal{X}_{1}$. In particular, $\mathcal{X}=\mathcal{X}_{1}$, $\mathcal{X}_{2}$.

Proof The first assertion is clear. By [1, Theorem II.9.3], the equations on $A_{i}$ $\left([i] \in \mathcal{P}_{1}\right)$ hold. Let $\sim$ be the relation on $\{0,1, \ldots, d\}$ defined as $i \sim j$ if $p_{j \alpha}^{i} \neq 0$ for some $\alpha \in[\alpha] \in \mathcal{P}_{1}$. Then $\sim$ is an equivalence relation. Clearly, $\{0\} \cup\left\{i:[i] \in \mathcal{P}_{1}\right\}$ is an equivalence class. For $[i] \in \mathcal{P}_{2}, p_{\alpha i}^{i} \neq 0$ for all $\alpha \in[\alpha] \in \mathcal{P}_{1}$. Since $i \neq \alpha$, by (B), $p_{\alpha j}^{i}=0$ if $j \neq i$. In particular, for $[j] \in \mathcal{P}_{2}, p_{j \alpha}^{i}=p_{\alpha j}^{i}=0$ if $j \neq i$. Hence each $i\left([i] \in \mathcal{P}_{2}\right)$ forms an equivalence class. By [1, Theorem II.9.4], the equations on $A_{i}$ $\left([i] \in \mathcal{P}_{2}\right)$ hold. The last assertion is clear by Definition 6.

Corollary 21 We keep the notation of Lemma 20. Both $\mathcal{X}_{1}$ and $\mathcal{X}_{2}$ are commutative association schemes whose intersection numbers satisfy (B) and whose corresponding posets are $\mathcal{P}_{1}$ and $\mathcal{P}_{2}$ respectively.

For details of imprimitive association schemes, see [1, Sect. II.9].

Lemma 22 Suppose $[i],[j] \in \mathcal{P}$ are incomparable. If $[i] \prec[h]$ for some $[h] \in \mathcal{P} \backslash$ $\{[i],[j]\}$, then $[j] \prec[h]$.

Proof Let $x \in X$. Take $y \in R_{i}(x), z \in R_{j}(x)$ and $w \in R_{h}(x)$. Since $[i] \prec[h]$, i.e., $p_{i h}^{h} \neq 0$, by (B), $p_{i l}^{h}=0$ if $l \neq h$. So $(y, w) \in R_{h}$. Let $(y, z) \in R_{s}$ and $(z, w) \in R_{t}$. By the configurations of 3 vertices $x, z, w$ and $y, z, w$, we obtain $p_{j t}^{h}=p_{t j}^{h} \neq 0$ and $p_{s t}^{h}=p_{t s}^{h} \neq 0$. If $t \neq h$, by (B), we have $s=j$ and by the configuration of $x, y, z$, we have $p_{i j}^{j} \neq 0$ contradicting the assumption that $[i],[j]$ are incomparable. Hence $t=h$ and $p_{j h}^{h} \neq 0$, i.e., $[j] \prec[h]$.

Proposition $23 \mathcal{P}$ is an ordinal sum of singletons and antichains.

Proof Let $\mathcal{P}_{1}$ be the set of minimal elements of $\mathcal{P}$. Clearly, $\mathcal{P}_{1}$ is either a singleton or an antichain. By Lemma $22, \mathcal{P}=\mathcal{P}_{1} \oplus\left(\mathcal{P} \backslash \mathcal{P}_{1}\right)$. Next let $\mathcal{P}_{2}$ be the set of minimal elements of $\mathcal{P} \backslash \mathcal{P}_{1}$. Similarly we obtain $\mathcal{P} \backslash \mathcal{P}_{1}=\mathcal{P}_{2} \oplus\left(\mathcal{P} \backslash\left(\mathcal{P}_{1} \oplus \mathcal{P}_{2}\right)\right)$. Repeating this process, we finally obtain $\mathcal{P}=\mathcal{P}_{1} \oplus \mathcal{P}_{2} \oplus \cdots \oplus \mathcal{P}_{m}$, where $\mathcal{P}_{i}(1 \leq i \leq m)$ is either a singleton or an antichain.

Proof of Theorem 1 (iii) $\Leftrightarrow$ (v) By Proposition $23, \mathcal{P}=\mathcal{P}_{1} \oplus \mathcal{P}_{2} \oplus \cdots \oplus \mathcal{P}_{m}$, where $\mathcal{P}_{l}(1 \leq l \leq m)$ is a singleton or an antichain. By Lemma 20, the subposet $\mathcal{P}_{1}$ induces the subscheme $\mathcal{X}_{1}$ of $\mathcal{X}$, and $\mathcal{X}=\mathcal{X}_{1}$ 2 $\mathcal{Y}_{1}$ where $\mathcal{Y}_{1}$ denotes the quotient scheme $\mathcal{X} / \mathcal{X}_{1}$. By Corollary $21, \mathcal{Y}_{1}$ satisfies (B) and corresponds to the poset $\mathcal{P}_{2} \oplus \cdots \oplus \mathcal{P}_{m}$. Again, the subposet $\mathcal{P}_{2}$ induces the subscheme $\mathcal{X}_{2}$ of $\mathcal{Y}_{1}$, and $\mathcal{Y}_{1}=\mathcal{X}_{2}$ ? $\mathcal{Y}_{2}$ where $\mathcal{Y}_{2}$ denotes the quotient scheme $\mathcal{Y}_{1} / \mathcal{X}_{2}$. Repeating this process, we finally obtain $\mathcal{X}=$ $\mathcal{X}_{1}$, $\mathcal{X}_{2} 2 \cdots>\mathcal{X}_{m}$, where $\mathcal{X}_{l}(1 \leq l \leq m)$ corresponds to the poset $\mathcal{P}_{l}$. By Lemma 19, $\mathcal{X}_{l}$ is a 1-class association scheme or the group association scheme of a finite abelian group. This completes the proof. 
Remark Let $\mathcal{X}$ be a symmetric association scheme. Then $\mathcal{X}$ is a wreath product of 1 -class association schemes if and only if $\mathcal{P}$ is a chain.

Acknowledgements The author would like to thank Hajime Tanaka for valuable discussions and suggestions. She would also like to thank Paul Terwilliger for his careful reading of the manuscript.

\section{References}

1. Bannai, E., Ito, T.: Algebraic Combinatorics I. Benjamin/Cummings, Redwood City (1984)

2. Bannai, E., Munemasa, A.: The Terwilliger algebras of group association schemes. Kyushu J. Math. 49, 93-102 (1995)

3. Brouwer, A.E., Cohen, A.M., Neumaier, A.: Distance-Regular Graphs. Springer, Berlin, Heidelberg (1989)

4. Bhattacharyya, G., Song, S.-Y., Tanaka, R.: On Terwilliger algebras of wreath products of one-class association schemes. J. Algebr. Comb. 31, 455-466 (2010)

5. Jaeger, F.: On spin models, triply regular association schemes, and duality. J. Algebr. Comb. 4, 103-144 (1995)

6. Martin, W.J., Tanaka, H.: Commutative association schemes. Eur. J. Comb. 30, 1497-1525 (2009)

7. Munemasa, A.: An application of Terwilliger algebra. Unpublished preprint. Preprint found on: http://www.math.is.tohoku.ac.jp/ munemasa/unpublished.html

8. Stanley, R.P.: Enumerative Combinatorics, vol. I. Cambridge Univ. Press, Cambridge (2002)

9. Terwilliger, P.: The subconstituent algebra of an association scheme (Part I). J. Algebr. Comb. 1, 363388 (1992). (Part II; III), J. Algebr. Comb. 2, 73-103; 177-210 (1993) 